\title{
New Adaptive Method for IQ Imbalance Compensation of Quadrature Modulators in Predistortion Systems
}

\author{
Hassan Zareian and Vahid Tabataba Vakili \\ Electrical Engineering Department, Iran University of Science and Technology (IUST), Tehran 16846-13114, Iran \\ Correspondence should be addressed to Hassan Zareian, zareian@ee.iust.ac.ir
}

Received 7 February 2009; Revised 13 May 2009; Accepted 23 June 2009

Recommended by George Tombras

\begin{abstract}
Imperfections in quadrature modulators $(\mathrm{QMs})$, such as inphase and quadrature (IQ) imbalance, can severely impact the performance of power amplifier (PA) linearization systems, in particular in adaptive digital predistorters (PDs). In this paper, we first analyze the effect of IQ imbalance on the performance of a memory orthogonal polynomials predistorter (MOP PD), and then we propose a new adaptive algorithm to estimate and compensate the unknown IQ imbalance in QM. Unlike previous compensation techniques, the proposed method was capable of online IQ imbalance compensation with faster convergence, and no special calibration or training signals were needed. The effectiveness of the proposed IQ imbalance compensator was validated by simulations. The results clearly show the performance of the MOP PD to be enhanced significantly by adding the proposed IQ imbalance compensator.
\end{abstract}

Copyright ( $\odot 2009$ H. Zareian and V. T. Vakili. This is an open access article distributed under the Creative Commons Attribution License, which permits unrestricted use, distribution, and reproduction in any medium, provided the original work is properly cited.

\section{Introduction}

High data rate wireless communications systems use spectrally efficient modulations such as the orthogonal frequency division multiplexing (OFDM). These systems are highly sensitive to the nonlinear distortion introduced by the power amplifier (PA) due to its nonconstant envelope and high peak-to-average ratio (PAR) values [1]. Therefore, one of the most challenging issues in designing PAs is the linearity requirement. The linearization techniques improve the linearity of PAs, and among them digital predistortion has gained considerable attention in recent years because of its low cost and reduced complexity [2].

A digital baseband predistortion system uses a quadrature modulator $(\mathrm{QM})$ to transfer the predistorted baseband signal to the RF. However, digital predistorter (PD) performance results are degraded by the IQ imbalance of QM in the direct up-conversion transmitter chain [3]. Therefore, the effects of IQ imbalance must be compensated, for example, by using digital signal processing.

The effect of IQ imbalance in QM on the complex gain PD with a look-up table (LUT) and the resulting performance degradation have been previously noted in [3].
To date, several techniques have been proposed to estimate and compensate the QM imperfections in direct-conversion transmitters [4-11]. The method presented in [4] considers a digital postcorrection at the receiver side. To estimate and compensate the IQ imbalance, some methods rely on special training signals [5-7]. The QM imperfections alone are compensated with an adaptive least mean square (LMS) algorithm in [8]. In [9], a two-step compensation approach is proposed, where first the QMC parameters are adjusted by bypassing the PA and then the PD parameters are found. The method in [10], based on minimization of out-of-band power, jointly compensates amplifier nonlinearity and QM errors. An adaptive technique that jointly compensates the PA nonlinearity and QM errors and does not need any extra feedback loop for QM/QDM error compensation is proposed in [11].

To the authors' knowledge, only a few previously published reports have considered IQ imbalance compensation in predistortion linearization systems [5, 7, 9-11]. The drawbacks of the existing compensation techniques are the following: (1) offline training using special calibration signals is needed $[5,7]$, (2) interruption of the regular transmission mode is required and generally, time-varying 


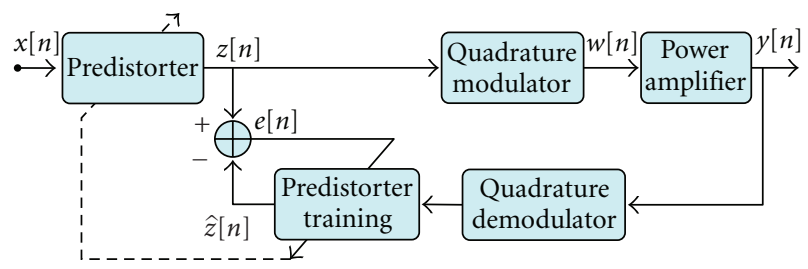

FIGURE 1: Block diagram of a direct-conversion transmitter with a predistortion linearization system.

QM errors, such as those caused by temperature variation, are impossible to track [9], (3) the convergence speed is very slow and the linearization performance of the PDs is not good [10], and (4) high computational and implementation complexity is involved in [11], and its application of the proposed compensation method is limited to use with the polynomial PDs. The proposed method in this paper solves these drawbacks as follows: (1) no special calibration training signals are needed, (2) the online calibration technique using only the regular transmit signal is capable of following the changes in the IQ imbalance parameters, (3) by using the recursive least squares (RLSs) adaptive algorithm the achieved convergence is faster as well as computationally more economical, and (4) it is not dependent on the predistortion systems and can be used in conjunction with all kinds of PDs. Moreover, the linearization performance of PDs can be significantly improved.

In summary, the contributions of this paper are the following. First, the effects of IQ imbalance in QM on the performance parameters of a memory orthogonal polynomials predistorter (MOP PD), such as the convergence behavior of PD coefficients adaptation, input amplitude to output amplitude (AM/AM) and input amplitude to input-output phase difference $(\mathrm{AM} / \mathrm{PM})$ characteristics of the linearized $\mathrm{PA}$, adjacent channel power ratio (ACPR), and error vector magnitude (EVM), were investigated. Second, an adaptive IQ imbalance compensator to enhance the PA linearization system performance is proposed.

The remainder of this paper is organized as follows. Section 2 briefly describes the problem of IQ imbalance and the predistortion compensation scheme. In this section we also introduce a recursive algorithm based on the MOP PD as the adaptive linearization technique for the memory PA. In Section 3, we study the influence of this QM imperfection on the MOP PD performance using simulation. To alleviate the IQ imbalance, we propose an adaptive digital algorithm for a QM compensator (QMC) in Section 4. Section 5 presents computer simulation results in order to evaluate the MOP $\mathrm{PD}$ performance in conjunction with the proposed IQ imbalance compensator. Finally, conclusions are given in Section 6.

Throughout the paper, the transpose and conjugate operations are denoted by $(\cdot)^{T}$ and $(\cdot)^{*}$, respectively.

\section{System Overview}

A simplified block diagram of a direct conversion transmitter with a predistortion linearization system is presented in
Figure 1. Note that the digital-to-analog converter (DAC), the reconstruction filter in feedforward path, as well as the antialiasing filter and the analog-to-digital converter (ADC) in the feedback path were ignored for simplicity. Moreover, all signals were treated as complex baseband.

The baseband input first goes through the PD, the output distorted signal of which is converted into an analog signal by the DAC and further up-converted in a QM to the RF frequency. Then, the signal is amplified by PA and transmitted. The feedback path is used for PD training. Since we are interested in a digital baseband PD, the passband PA output is translated to its equivalent digital baseband form using the quadrature demodulator (QDM) and ADC.

Unfortunately, there are imperfections, such as the IQ imbalance in the analog QM and QDM, which can affect the PD performance greatly [12]. In this paper, we assumed an ideal QDM to form the complex baseband signal, and the IQ imbalance in QM was assumed to be invariant with frequency.

2.1. IQ Imbalance Compensation. Let $g_{m}$ and $\varphi_{m}$ denote gain and phase imbalances between the in-phase (I) and quadrature $(\mathrm{Q})$ branches in the $\mathrm{QM}$, respectively. Using matrix notation, the distortions caused by the IQ imbalance in QM can be modeled by [13]

$$
\left[\begin{array}{l}
w_{I}[n] \\
w_{Q}[n]
\end{array}\right]=\mathbf{m}\left[\begin{array}{c}
z_{I}[n] \\
z_{Q}[n]
\end{array}\right], \quad \mathbf{m}=\left[\begin{array}{cc}
1 & -g_{m} \sin \left(\varphi_{m}\right) \\
0 & g_{m} \cos \left(\varphi_{m}\right)
\end{array}\right],
$$

where $w[n]=w_{I}[n]+j w_{Q}[n]$ is the complex baseband representation of the QM's output distorted signal. The terms $z_{I}[n]$ and $z_{Q}[n]$ are the inphase and quadrature components of the baseband signal of $z[n]$, respectively.

The IQ imbalance compensation based on digital predistortion is more convenient, less expensive, and more precise to implement. As shown in Figure 2, having obtained the estimates of the gain and phase imbalances, that is, $\hat{g}_{m}$ and $\hat{\varphi}_{m}$, respectively, we use them to compensate the $z_{I}[n]$ and $z_{Q}[n]$ baseband signals before applying them to the QM. Therefore, we can obtain the compensated $u_{I}[n]$ and $u_{Q}[n]$ baseband signals by

$$
\left[\begin{array}{l}
u_{I}[n] \\
u_{Q}[n]
\end{array}\right]=\mathbf{c}\left[\begin{array}{l}
z_{I}[n] \\
z_{Q}[n]
\end{array}\right], \quad \mathbf{c}=\mathbf{m}^{-1}=\left[\begin{array}{cc}
1 & \tan \left(\hat{\varphi}_{m}\right) \\
0 & \frac{1}{\widehat{g}_{m} \cos \left(\hat{\varphi}_{m}\right)}
\end{array}\right] .
$$




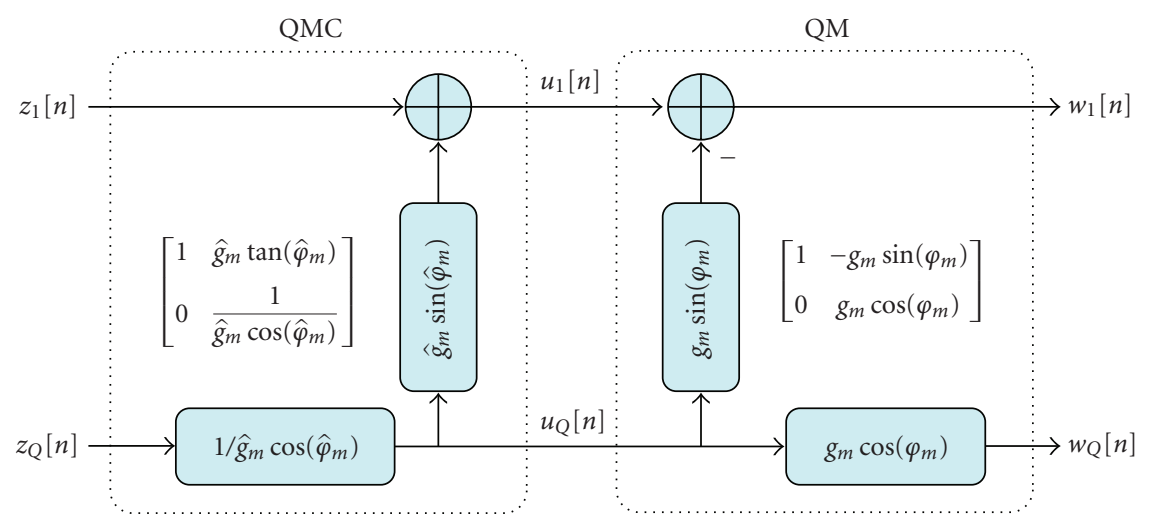

FIGURE 2: QM baseband model and digital predistortion structure of QMC.

Perfect compensation $(w[n]=z[n])$ can be achieved if the parameters of the QMC are equal to the real IQ imbalance parameters of the $\mathrm{QM}\left(\hat{\varphi}_{m}=\varphi_{m}, \hat{g}_{m}=g_{m}\right)$.

Since $g_{m}$ and $\varphi_{m}$ are unknown in practice, they have to be estimated. In this paper a new IQ imbalance compensation scheme is presented, in which the unknown analog imbalance parameters are estimated digitally, without the need for any calibration or training signal.

2.2. An Adaptive $M O P P D$. In this section, we introduce an adaptive identification algorithm for the MOP PD with an indirect learning structure, as shown in Figure 1. The benefit of this structure is that we can obtain directly the PD parameters instead of assuming a model for the PA, estimating the PA parameters, and then constructing its inverse [14].

In the absence of IQ imbalance, we would have $w[n]=$ $z[n]$. Ideally, the output of this system should be $y[n]=x[n]$, when $z[n]$ equals $\hat{z}[n]$; that is, it makes the error term $e[n]=$ $\hat{z}[n]-z[n]=0$. The output-input relation of the PD training block can be described by a memory orthogonal polynomial (MOP) model [15]:

$$
z[n]=\sum_{\substack{k=1 \\ \text { Odd }}}^{K} \sum_{q=0}^{Q} \alpha_{k q} \psi_{k q}[n],
$$

where the memory length of the PD and the polynomial order are equal to $Q$ and $K$, respectively, and $\alpha_{k q}$ are complexvalue coefficients. The memory orthogonal polynomial basis function $\psi_{k q}[n]$ for a complex Gaussian process with zero mean and $\sigma_{y}^{2}$ variance, such as an OFDM signal, is defined $[16]$ as

$$
\psi_{2 m+1, q}[n]=\sum_{k=0}^{m} \frac{(-1)^{m-k}}{\sigma_{y}^{2 k+1}} \frac{\sqrt{m+1}}{(k+1) !}\left(\begin{array}{c}
m \\
k
\end{array}\right) \phi_{2 k+1, q}[n],
$$

where $\phi_{k q}[n] \triangleq|y[n-q]|^{k-1} y[n-q]$ is the conventional memory polynomial basis function. It is noted that $y[n]$ is non-Gaussian, before identification and predistortion, even if $x[n]$ is Gaussian. However, the orthogonal polynomials can alleviate the numerical instability problem associated with the conventional polynomials even if the signal distribution deviates from complex Gaussian [16].

We used an adaptive approach to obtain the predistorter coefficients. In the remainder of this paper the predistorter based on this solution will be called MOP PD. MOP PD employs the RLS adaptive algorithm to track timevarying PA characteristics caused by changes in operating temperature, component aging, and environmental and manual variations. Therefore, adaptation of the predistorter was achieved by comparing $\hat{z}[n]$ with $z[n]$ and adjusting the predistorter parameters $\alpha_{k q}$ by minimizing the criterion:

$$
J[n]=\sum_{l=1}^{n} \lambda^{n-l}|e[l]|^{2}=\sum_{l=1}^{n} \lambda^{n-l}|z[l]-\widehat{z}[l]|^{2},
$$

where $\lambda$ is the forgetting factor. Using matrix notation we can reformulate (5) as

$$
z[n]=\boldsymbol{\alpha}^{T} \psi[n],
$$

where

$$
\begin{gathered}
\boldsymbol{\alpha}=\left[\alpha_{10}, \ldots, \alpha_{K 0}, \ldots, \alpha_{1 Q} \ldots, \alpha_{K Q}\right]^{T}, \\
\psi[n]=\left[\psi_{10}[n], \ldots, \psi_{K 0}[n], \ldots, \psi_{1 Q}[n], \ldots, \psi_{K Q}[n]\right]^{T} .
\end{gathered}
$$

The RLS adaptive algorithm to extract the predistorter coefficient vector $\alpha$ is initialized [17] by

$$
\begin{gathered}
\mathbf{P}[0]=\delta^{-1} \mathbf{I}, \\
\alpha[0]=[0.1,0, \ldots, 0]^{T},
\end{gathered}
$$

where $\delta$ is a small positive constant, $\alpha[0]$ is a $K^{\prime}(Q+1)$ column vector, where $K^{\prime}$ is $(K+1) / 2$. In order to update the coefficient vector of $\boldsymbol{\alpha}$ at every iteration, $n=1,2, \ldots$, the calculation of the following equations is required

$$
\begin{aligned}
e[n] & =z[n]-\hat{z}[n]=z[n]-\boldsymbol{\alpha}^{T}[n-1] \psi[n], \\
\mathbf{K}[n] & =\frac{\mathbf{P}[n-1] \boldsymbol{\psi}^{*}[n]}{\lambda+\psi^{T}[n] \mathbf{P}[n-1] \boldsymbol{\psi}^{*}[n]}, \\
\boldsymbol{\alpha}[n] & =\boldsymbol{\alpha}[n-1]+\mathbf{K}[n] e[n], \\
\mathbf{P}[n] & =\frac{1}{\lambda} \mathbf{P}[n-1]-\frac{1}{\lambda} \mathbf{K}[n] \psi^{T}[n] \mathbf{P}[n-1]
\end{aligned}
$$




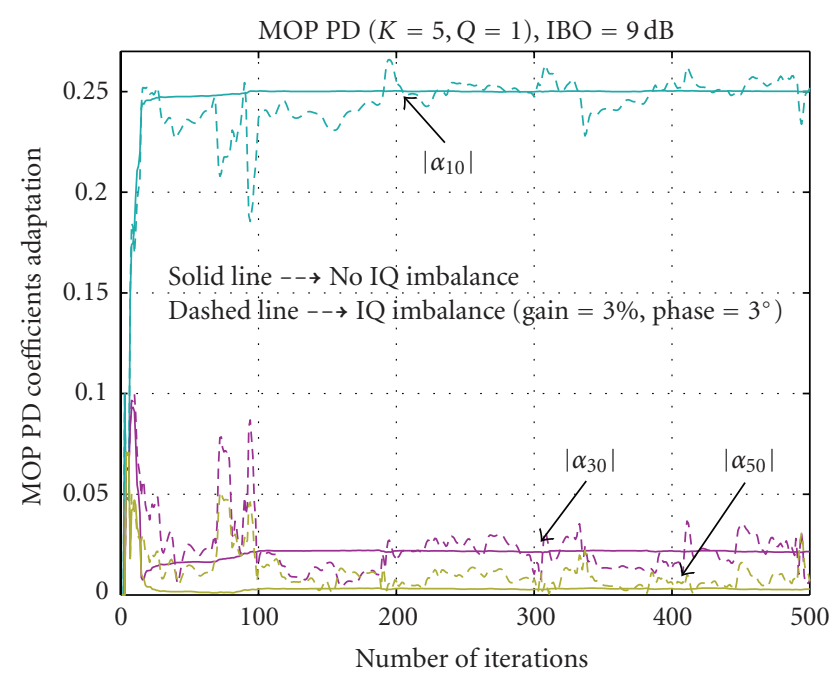

FIgURE 3: Convergence behavior of the MOP PD coefficients adaptation with and without IQ imbalance.

\section{Effects of IQ Imbalance in QM on MOP PD Performance}

The effect of IQ imbalance on the linearization performance of the MOP PD is presented in this section. We have carried out system simulations based on equivalent models to evaluate the linearization performance of the MOP PD in the presence of IQ imbalance in terms of convergence behavior, $\mathrm{AM} / \mathrm{AM}$, and $\mathrm{AM} / \mathrm{PM}$ curves and EVM and ACPR performances.

The performance of the proposed scheme was evaluated using the $2 \mathrm{~K}$-mode OFDM signal based on the European digital video broadcasting terrestrial (DVB-T) standard [18]. In addition, the PA with memory effect was assumed to obey a Wiener model, implemented as a 3-tap FIR filter with the coefficients [0.7692, 0.1538, 0.0769] [19], followed by a Saleh TWTA model [20]. The operating point of the PA is usually identified by the back-off. The input back-off (IBO) and the output back-off $(\mathrm{OBO})$ are defined as

$$
\begin{aligned}
& \mathrm{IBO}=10 \log \frac{A_{s}^{2}}{\sigma_{x}^{2}}, \\
& \mathrm{OBO}=10 \log \frac{A_{o}^{2}}{\sigma_{y}^{2}}
\end{aligned}
$$

where $A_{s}^{2}$ denotes the input saturation power of the PA, and $A_{o}^{2}$ presents the maximum PA output power. Moreover, $\sigma_{x}^{2}$ and $\sigma_{y}^{2}$ are the mean power of the input and output signals, respectively. Throughout the simulations, the IBO was chosen as $9 \mathrm{~dB}$, which is equivalent to an $\mathrm{OBO}$ of $4.7 \mathrm{~dB}$. We have set the parameters of the MOP PD to $K=5$ and $Q=1$ for the order of orthogonal polynomial and length of memory, respectively. Moreover, we chose a forgetting factor of $\lambda=0.95$ and used an oversampling factor of 8 for simulations.
Convergence properties were analyzed through RLS coefficients behavior. As shown in Figure 3, the IQ imbalance affects the convergence behavior of the MOP PD coefficients adaptation and caused the coefficients of the system estimator not to converge to their respective optimum values.

Figure 4 gives the variation histogram of the first MOP PD coefficient $\left(\alpha_{10}\right)$ during the adaptation algorithm for several cases. In addition to the ideal IQ balance case, the following two IQ imbalance cases were considered: case A, with $g_{m}=1.03, \varphi_{m}=3^{\circ}$ and case B, with $g_{m}=1.1, \varphi_{m}=10^{\circ}$. From this figure, it could be concluded that the variance of $\alpha_{10}$ in the presence of IQ imbalance has increased.

The influence of IQ imbalance on the linearization performance of the MOP PD can be checked out by comparing the $\mathrm{AM} / \mathrm{AM}$ and $\mathrm{AM} / \mathrm{PM}$ relationship between the input and output of the linearized PA. This will demonstrate the IQ imbalance effect on the accuracy of the MOP PD in linearizing the PA and increasing memory effects by thickening the curves.

The AM/AM and AM/PM curves obtained with predistortion for the three IQ imbalance cases are shown in Fig. 5. We note that without IQ imbalance, the AM/AM and $\mathrm{AM} / \mathrm{PM}$ curves were thinner, and the output amplitude has been thoroughly linearized. We can also use the normalized mean square error (NMSE) as a figure of merit to measure the linearity of the power amplifier, where it is defined by

$$
\text { NMSE }=10 \log _{10}\left(\frac{\sum_{k}|x[k]-y[k]|^{2}}{\sum_{k}|x[k]|^{2}}\right),
$$

where $y[n]$ is the linearized PA baseband output and, $x[n]$ is its corresponding input (desired output). The measured results of NMSE are shown in Figure 5, where the NMSE performance of the linearized PA was degraded by 11 and $20 \mathrm{~dB}$ for cases $\mathrm{A}$ and $\mathrm{B}$, respectively, in comparison with an ideal IQ balance case.

In the following, we evaluate the EVM performance of the MOP PD in the presence of IQ imbalance in QM. The EVM criterion is of fundamental importance as it is linked with the symbol error rate (SER) or bit error rate (BER) performance. We used a constellation diagram to quantify the deformation of the constellation at the output of the PA with an EVM parameter defined by:

$$
\mathrm{EVM}=\sqrt{\frac{\min _{r_{0}, r_{1}} \sum_{k}\left|d_{x}[k]-\left(\left(d_{y}[k]-r_{1}\right) / r_{0}\right)\right|^{2}}{\sum_{k}\left|d_{x}[k]\right|^{2}}},
$$

where $d_{x}[k]$ and $d_{y}[k]$, respectively, denote a sequence of $16-$ QAM-OFDM signals and their corresponding demodulated values. The parameters $r_{0}$ and $r_{1}$ were optimized to compensate for rotation and offset of the constellation.

Figure 6 shows the effects of IQ imbalance on the 16QAM-OFDM constellation at the linearized PA output for the differential values of gain and phase imbalances as well as its corresponding EVM value. This result indicates that IQ imbalances have a large impact on the EVM performance of the MOP PD. Specifically, the EVM increased from about 


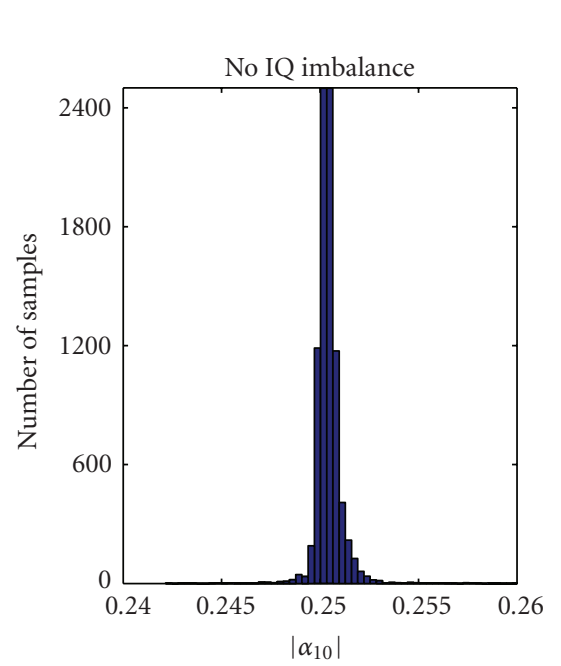

(a)

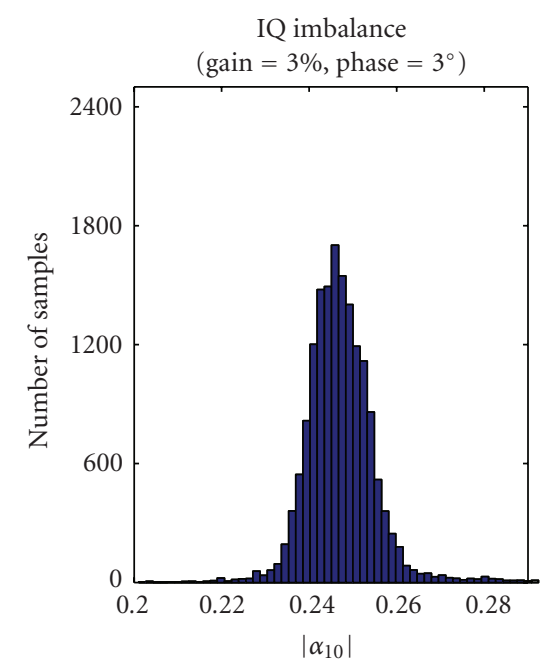

(b)

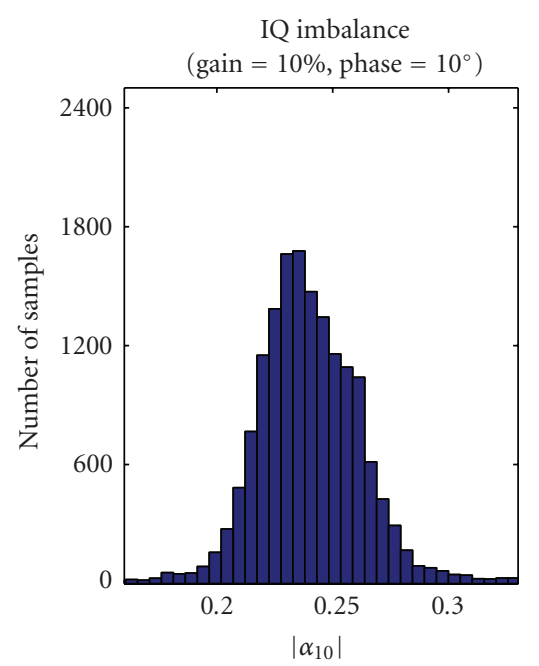

(c)

FIgURE 4: The variation histogram of the first MOP PD coefficient with and without IQ imbalance.

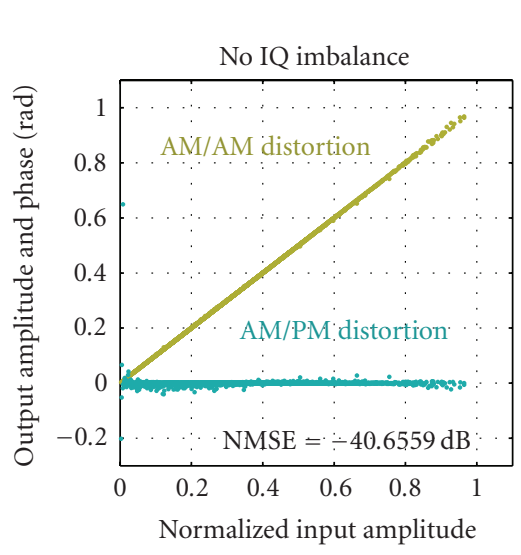

(a)

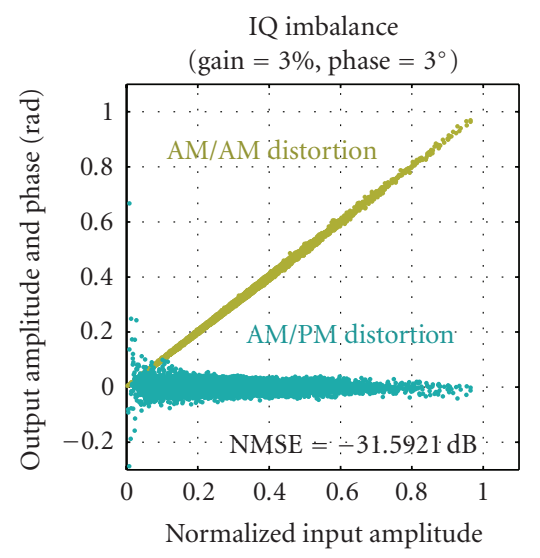

(b)

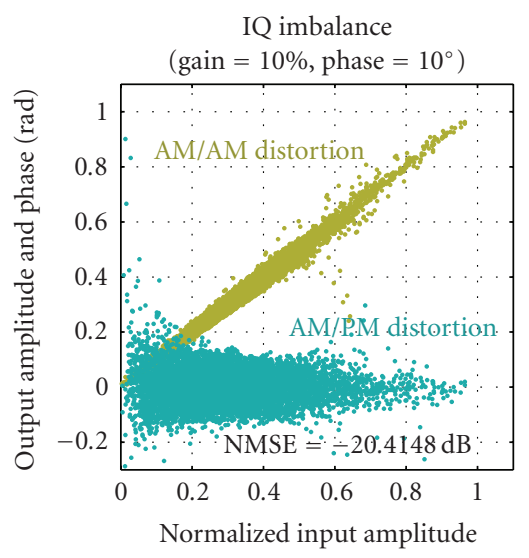

(c)

FIgURE 5: The AM/AM and AM/PM curves of the linearized PA with and without IQ imbalance.

$0.7 \%$ without IQ imbalance to approximately $2.2 \%$ and $8.1 \%$ for cases A and B, respectively.

Finally, to qualify the effect of IQ imbalance on the ACPR performance of the MOP PD, we analyzed the power spectral densities (PSDs) of the input and output signals of the linearized PA. The ACPR characterizes the spectral regrowth and is defined as the ratio of power radiated into an adjacent channel to the power in the main channel:

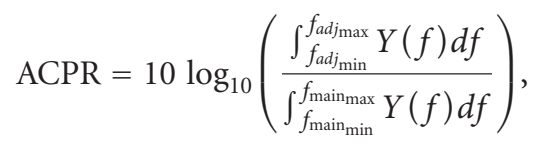

where $f$ denotes the frequency and $Y(f)$ the PSD of the PA output signal. The normalized PSDs of the output signal and the corresponding ACPR values computed by (13) were shown in Figure 7. For comparison, the PSD of the original input signal and the output PSD of the system employing an ideal PD without IQ imbalance also are shown. As noted before, the IQ imbalance in QM can dramatically degrade the ACPR performance of the MOP PD. For instance, the resulting $A C P R$ values for case $A(-41 \mathrm{~dB})$ and $B(-32 \mathrm{~dB})$ are larger than without IQ imbalance $(-52 \mathrm{~dB})$.

This section illustrated the effect of IQ imbalance on the MOP PD performance and the need for a method to achieve IQ imbalance compensation, which will be presented in the next section.

\section{Proposed IQ Imbalance Compensation}

A complete block diagram of the system model for the proposed IQ compensator in conjunction with the MOP PD is shown in Figure 8.

Based on the baseband input $u[n]$ and the passband output of the QM, we developed a method to estimate the QM model parameters and construct the associated QMC by the digital predistortion technique (see Figure 2). An inverse memory PA model (the MOP PD) is also estimated using the RLS algorithm to achieve PA linearization. 


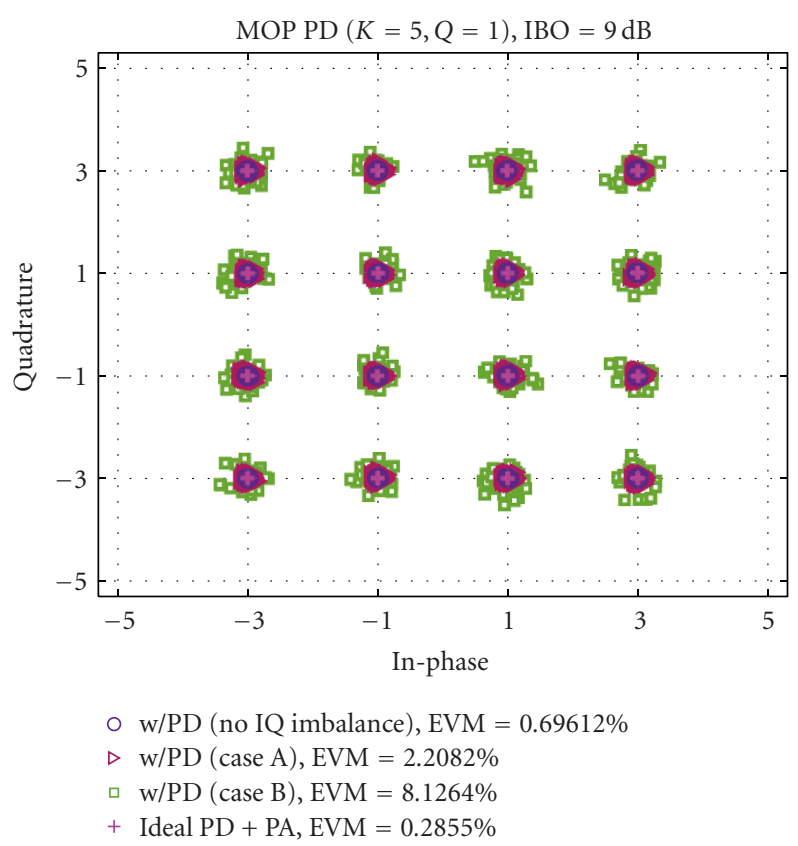

FIGURE 6: 16-QAM-OFDM constellation and the corresponding EVM value of simulated output of the linearized PA with and without IQ imbalance.

Two parameters, $g_{m}$ and $\varphi_{m}$, are to be estimated using any algorithm, depending on the desired performance in the asymmetric model of Figure 2, with the LMS and RLS algorithms being the most obvious ones resulting in different convergence speeds and computational complexities. The RLS algorithm was used in this paper to provide fast convergence [17].

The actual signal applied to the PA was a bandpass signal with a complex envelope produced by the QM, which is itself preceded by the QMC.

The ideal envelope detector (ED) in Figure 8 can be characterized using an ideal square-law envelope detector followed by the ideal ADC as shown in Figure 9. The discrete output baseband signal $s[n]$ is related to the complex envelope of the RF input signal by

$$
s[n]=|w[n]|^{2} .
$$

In the following, we derived an RLS algorithm for estimation and compensation of the IQ imbalance based on the envelope of the output signal at QM. The adaptation of the RLS algorithm is to minimize the criterion J on the error $e_{\mathrm{qm}}[n]=s[n]-\widehat{s}[n]$,

$$
J[n]=\sum_{l=1}^{n} \lambda^{n-l}\left|e_{\mathrm{qm}}[l]\right|^{2},
$$

where $\lambda$ is the forgetting factor. We first rewrite (1) in linear form:

$$
\widehat{w}(t)=\left[z_{I}(t)-g_{m} \sin \left(\varphi_{m}\right) z_{Q}(t)\right]+j g_{m} \cos \left(\varphi_{m}\right) z_{Q}(t) .
$$

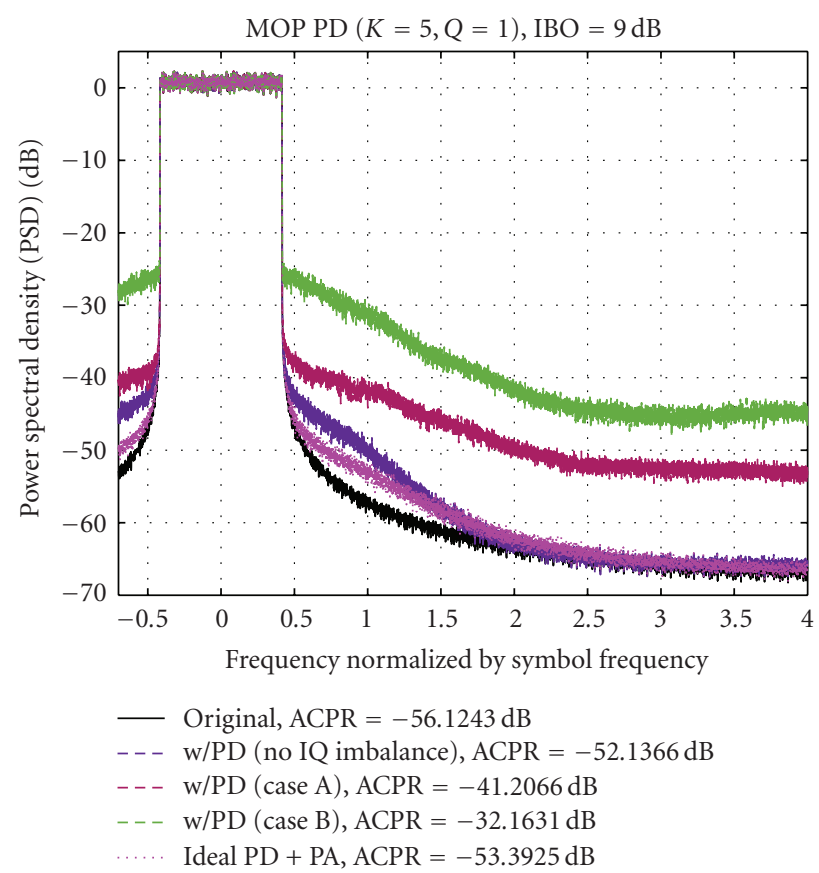

FIgURE 7: Normalized PSD and the corresponding ACPR value of the simulated input and output of the linearized PA with and without IQ imbalance.

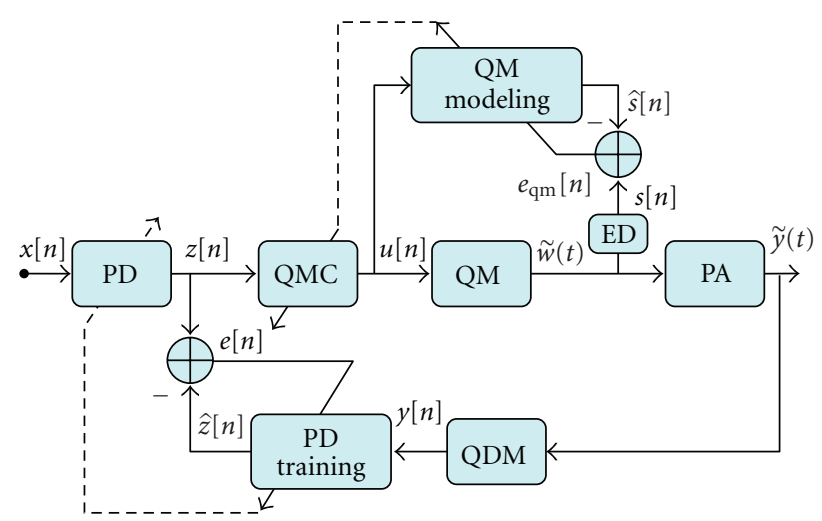

FIGURE 8: The proposed adaptive compensator structure in conjunction with the MOP PD.

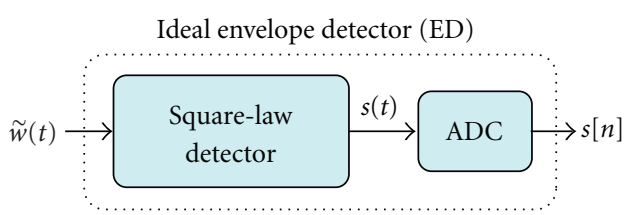

FIGURE 9: Block diagram of internal ideal ED structure.

By substituting (16) into (14) through some manipulations, (14) can be rewritten as

$$
\widehat{s}[n]-z_{I}^{2}[n]=\zeta^{T} \chi[n]
$$




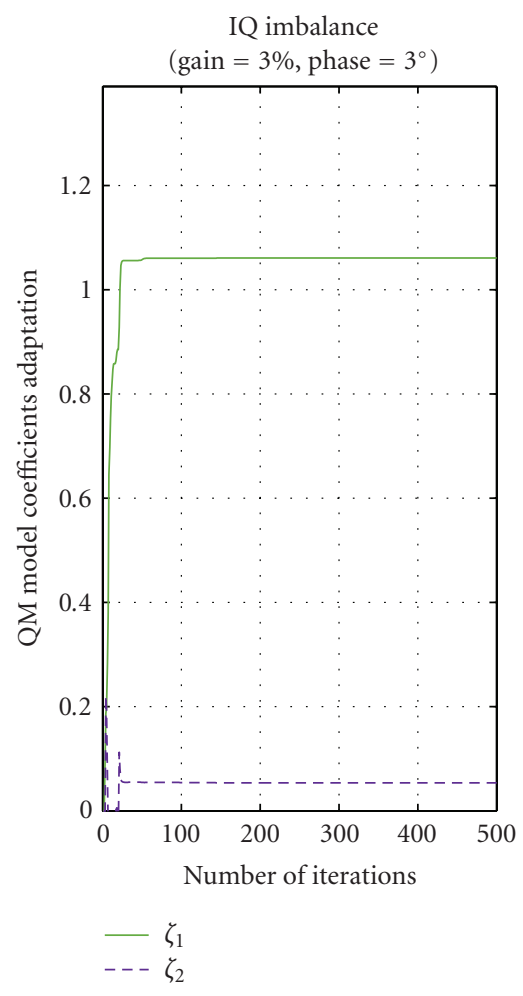

(a)

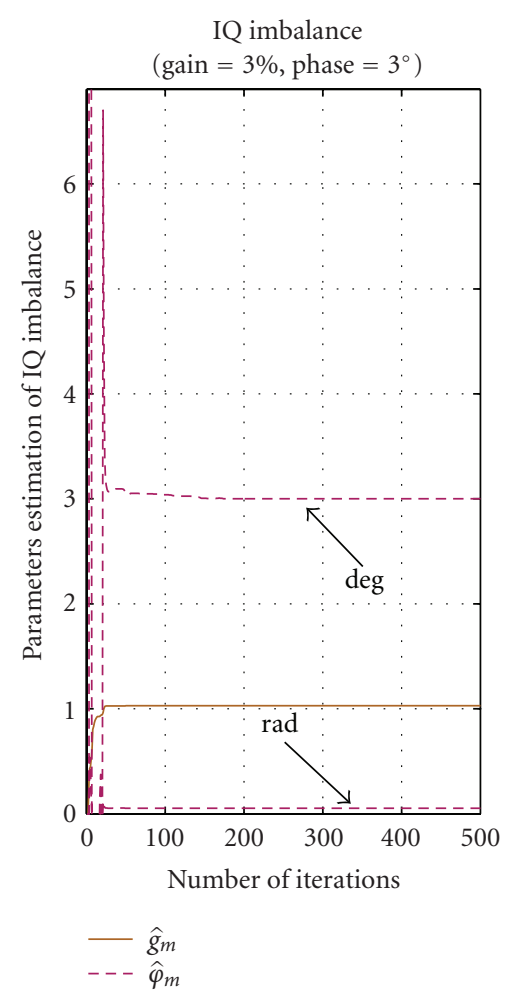

(b)

FIGURE 10: Convergence behavior of (a) QM model coefficients adaptation (b) the corresponding parameters estimation of IQ imbalance.

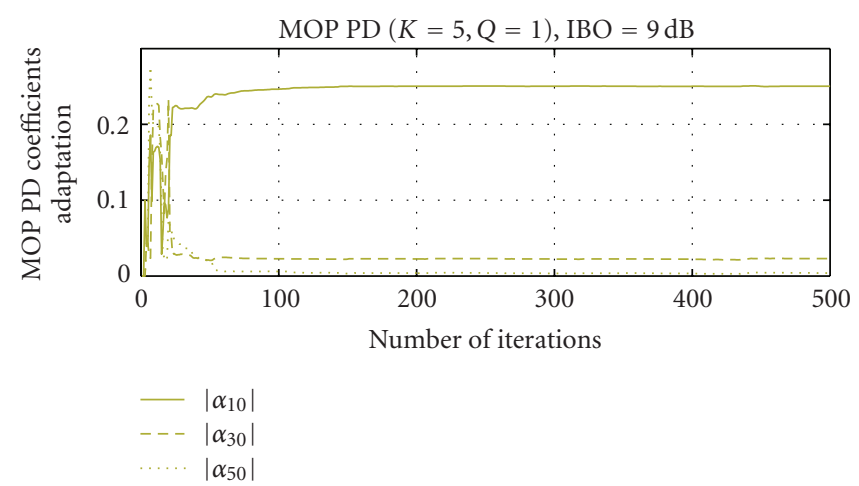

(a)

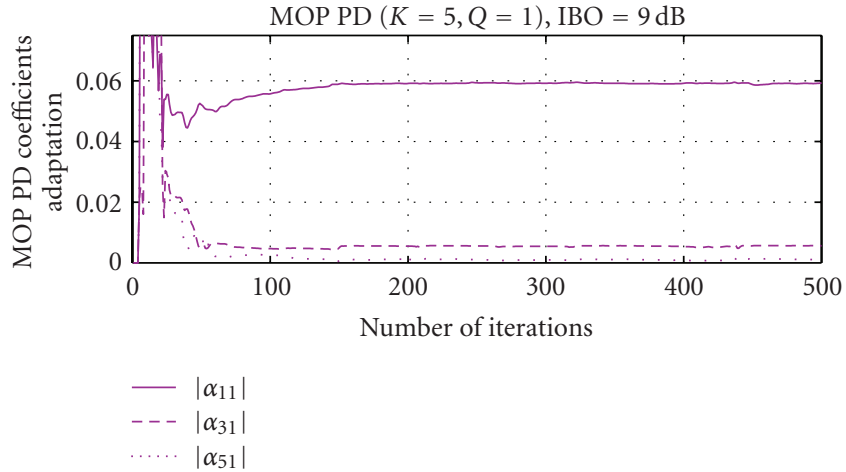

(b)

FIGURE 11: Convergence behavior of the MOP PD coefficients adaptation.

where $\zeta$ and $\chi[n]$ are complex vectors defined by

$$
\begin{gathered}
\zeta=\left[\zeta_{1}, \zeta_{2}\right]^{T}, \\
\chi[n]=\left[z_{Q}^{2}[n],-2 z_{I}[n] z_{Q}[n]\right]^{T} .
\end{gathered}
$$

The QM model coefficients of $\zeta_{1}$ and $\zeta_{2}$ are related to the gain and phase imbalances as follows:

$$
\begin{gathered}
\zeta_{1}=g_{m}^{2}, \\
\zeta_{2}=g_{m} \sin \left(\varphi_{m}\right) .
\end{gathered}
$$

Note that in order to obtain the parameters of the IQ imbalance in the asymmetric QM model, we have applied a two-step technique for each instant of $n$. During the first step, $\zeta_{1}$ and $\zeta_{2}$ were estimated, and for the second step, the $g_{m}$ and $\varphi_{m}$ were derived and reserved as the parameters of compensator for the next instant, $n+1$.

By defining $\bar{s}[l] \triangleq s[l]-z_{I}^{2}[l]$ and $\overline{\hat{s}}[l] \triangleq \hat{s}[l]-z_{I}^{2}[l]$, we can modify the criterion $J$ in (15) as

$$
J[n]=\sum_{l=1}^{n} \lambda^{n-l}|\bar{s}[l]-\overline{\hat{s}}[l]|^{2} .
$$




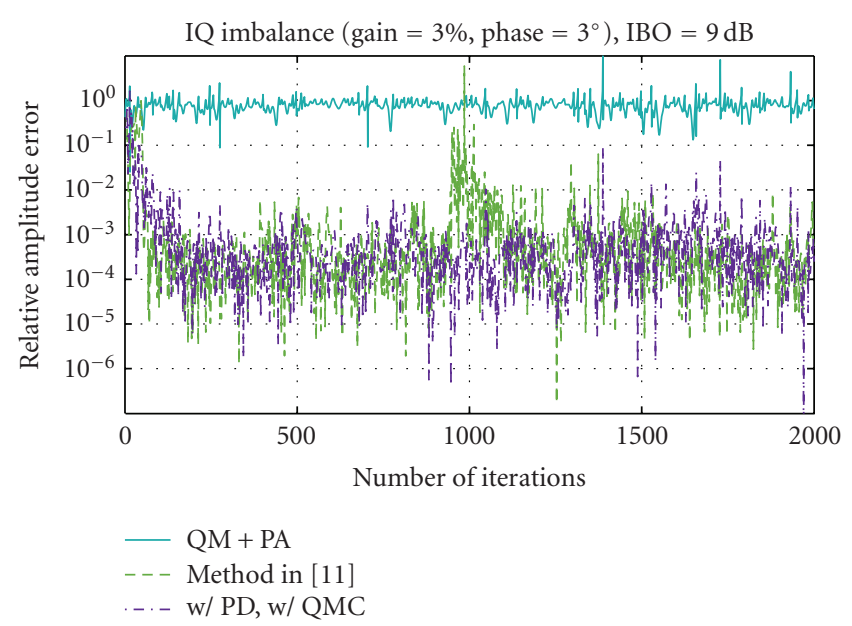

(a)

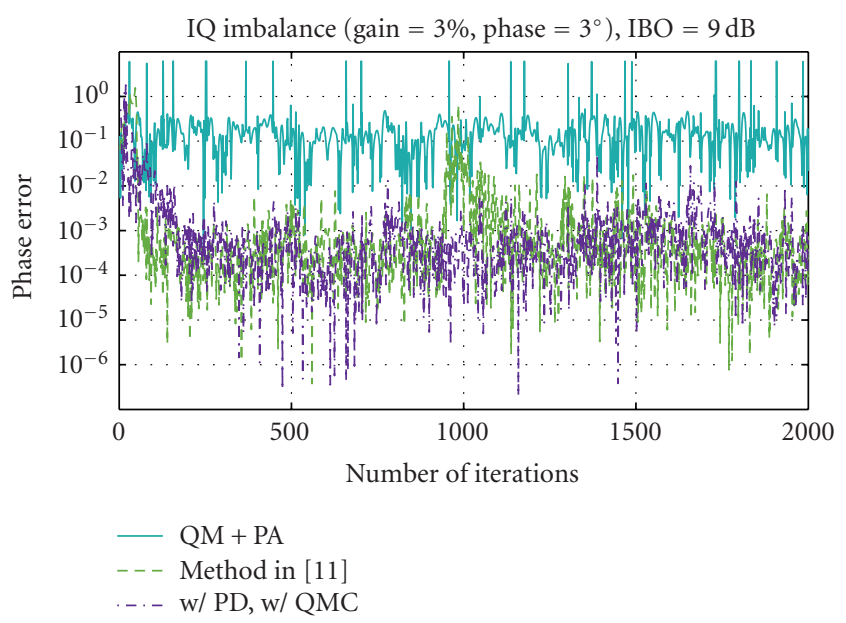

(b)

FIGURE 12: Instantaneous errors between the input and output of the linearized PA with the proposed IQ imbalance compensator (a) relative amplitude error (b) phase error.

Therefore, we have the following adaptive algorithm for updating $\zeta[n]$ for every $n=1,2, \ldots$, directly computed from (21)-(24):

$$
\begin{aligned}
e_{\mathrm{qm}}[n] & =\bar{s}[n]-\overline{\hat{s}}[l]=s[n]-z_{I}^{2}[n]-\zeta^{T}[n-1] \chi[n], \\
\mathbf{K}[n] & =\frac{\mathbf{P}[n-1] \chi^{*}[n]}{\lambda+\chi^{T}[n] \mathbf{P}[n-1] \chi^{*}[n]}, \\
\zeta[n] & =\zeta[n-1]+\mathbf{K}[n] e_{\mathrm{qm}}[n], \\
\mathbf{P}[n] & =\frac{1}{\lambda} \mathbf{P}[n-1]-\frac{1}{\lambda} \mathbf{K}[n] \chi^{T}[n] \mathbf{P}[n-1] .
\end{aligned}
$$

The RLS adaptive algorithm was initialized by setting

$$
\begin{gathered}
\mathbf{P}[0]=\delta^{-1} \mathbf{I}, \\
\zeta[0]=[0.1,0]^{T},
\end{gathered}
$$

where $\delta$ is a small positive constant. The IQ imbalance parameters calculated from (2) at instant $n$ were applied as the new values of proposed QMC for instant $(n+1)$ :

$$
\begin{gathered}
\hat{g}_{m}[n+1]=\sqrt{\zeta_{1}[n]}, \\
\hat{\varphi}_{m}[n+1]=\arcsin \left(\frac{\zeta_{2}[n]}{\sqrt{\zeta_{1}[n]}}\right) .
\end{gathered}
$$

\section{Simulation Results}

In this section, we evaluate the linearization performance of the MOP PD in conjunction with the proposed IQ imbalance compensator using simulation with parameters similar to those of Section 3. For both RLS algorithms, the forgetting factor we used was 0.95 . For comparison, we also considered another IQ imbalance compensation method introduced in [11], which jointly estimates the PD and QMC parameters.

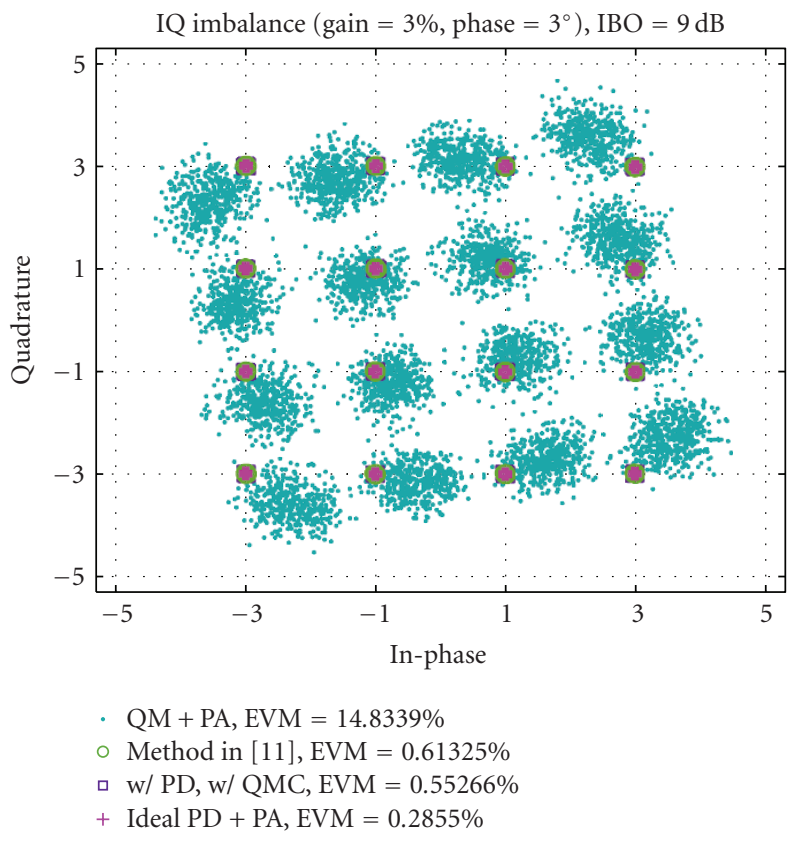

FIGURE 13: EVM performance of the MOP PD without and with the proposed QMC.

First, the convergence behavior of the proposed QMC and MOP PD adaptive algorithms was studied for an IQ imbalance case with $g_{m}=1.03$, and $\varphi_{m}=3^{\circ}$.

Figure 10 shows the convergence behavior of QM model coefficients adaptation, and the corresponding parameters estimation of IQ imbalance as a function of the number of iterations. According to these figures, it was clear that the merit of the proposed compensator was rapid convergence of parameters within fewer than just 100 samples. As shown in Figure 10(a), the estimated values of gain and phase imbalance were extremely near to the true values of the given 


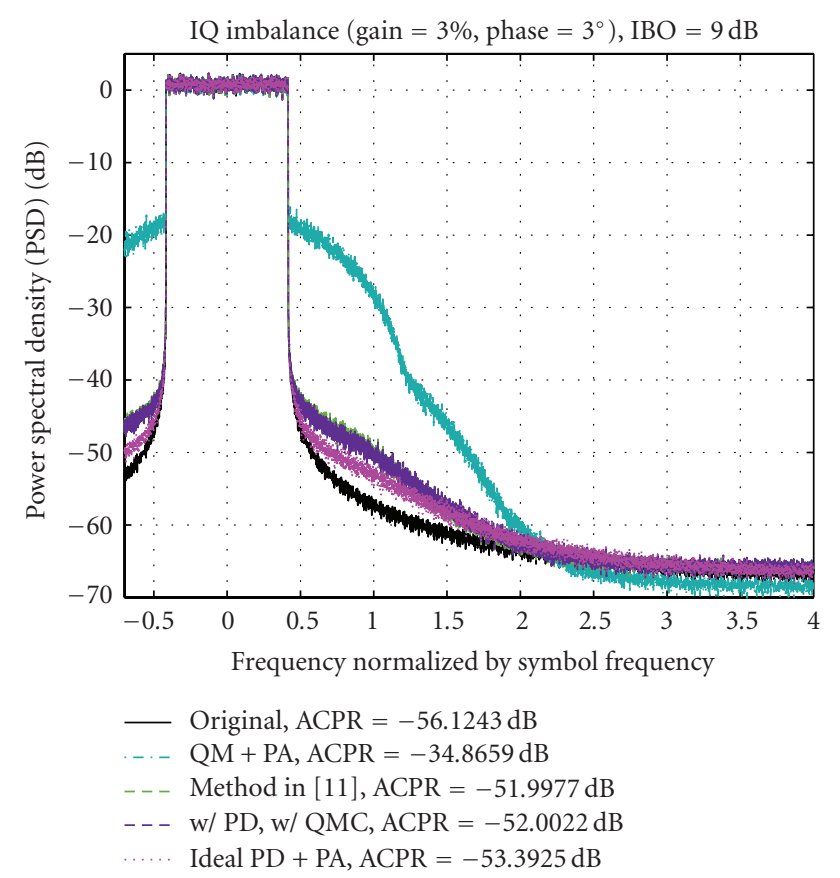

FIGURE 14: ACPR performance of the MOP PD with and without the proposed IQ imbalance compensator.

IQ imbalance parameters $\left(\hat{g}_{m}=1.03, \hat{\varphi}_{m}=3^{\circ}\right)$. The rapid and reliable convergence of the MOP PD coefficients toward their optimal values by adding the proposed adaptive IQ imbalance compensator is shown in Figure 11.

Figure 12 shows the instantaneous errors (amplitude and phase) as a function of the number of iterations, with and without a joint compensator. After convergence, both the proposed method and the method of [11] reduced the error significantly and showed fast convergence, but the proposed method was still somewhat more accurate and reliable than the method in [11].

To evaluate EVM performance of the MOP PD, the transmitted signal constellation with the proposed correction method is shown in Figure 13. For comparison, the EVM performance of the method in [11] and an ideal PD without IQ imbalance (i.e., soft limiter) are presented as well. It is noted that the EVM performance improves from about $2.2 \%$ (see Figure 6) to approximately $0.55 \%$ (slightly less than the method in [11]) after the proposed QMC method is applied, obtaining a residual vector error of less than $0.3 \%$ of the ideal case of linear amplification without IQ imbalance.

Figure 14 compares the normalized PSD of the output signal and the corresponding ACPR value for a linearized PA with and without the proposed adaptive correction method. From Figure 14, it is evident that with the proposed compensation techniques or method of [11], the ACPR performance improved from -41 (see Figure 7) to $-52 \mathrm{~dB}$, an improvement of about $11 \mathrm{~dB}$.

Based on the above discussion, the digital IQ imbalance compensator similar to the method in [11] was an effective technique for improving the linearization performance of the MOP PD. In the following, the complexity of both the proposed method and the method in [11] is evaluated. Although the proposed method used an extra loop for QM compensation that has only an envelope detector for adaptation, unlike the method in [11], it estimated the QMC parameters and the MOP PD coefficients directly, and the length of coefficients vector was deduced from $2 K^{\prime}(Q+$ $1)=12($ see $[11])$ to $K^{\prime}(Q+1)+2=8$ by using the proposed method. Consequently, the computational complexity and implementation of the proposed algorithm were quite modest.

The major advantage of the proposed method over that in [11] is its independence from the predistortion systems that can be used in conjunction with any variety of PDs.

\section{Conclusions}

In this paper, the effects of QM imperfections, such as gain and phase imbalances on the MOP PD performance, were examined through computer simulations. The results show that the MOP PD does not achieve sufficient compensation of the PA effects in the presence of IQ imbalance in QM. Therefore, we have proposed an adaptive algorithm to estimate and correct the IQ imbalance of QM and to enhance the MOP PD performance.

The proposed compensation technique was used in conjunction with the MOP PD performance, and using computer simulations we showed that the MOP PD achieves significant improvements over the case without any IQ imbalance compensation. For instance, with a gain imbalance of approximately 3\% and a phase imbalance of around $3^{\circ}$, the EVM and the ACPR performances of the MOP PD improved by $3 \%$ and $8 \mathrm{~dB}$, respectively, after applying the proposed correction method.

Unlike previously published techniques, the introduced adaptive method is capable of online IQ imbalance compensation without using special training signals. It presents faster adaptation and more reliable convergence than previously published methods, which enables it to track the possible variations of IQ imbalance parameters of QM.

\section{References}

[1] R. Van Nee and R. Prasad, OFDM for Wireless Multimedia Communications, Artech House, Norwood, Mass, USA, 2000.

[2] P. B. Kenington, High-Linearity RF Amplifier Design, Artech House, Norwood, Mass, USA, 2000.

[3] J. K. Cavers, "The effect of quadrature modulator and demodulator errors on adaptive digital predistorters for amplifier linearization," IEEE Transactions on Vehicular Technology, vol. 46, no. 2, pp. 456-466, 1997.

[4] X. Huang, "On transmitter gain/phase imbalance compensation at receiver," IEEE Communications Letters, vol. 4, no. 11, pp. 363-365, 2000.

[5] J. K. Cavers and M. W. Liao, "Adaptive compensation for imbalance and offset losses in direct conversion transceivers," IEEE Transactions on Vehicular Technology, vol. 42, no. 4, pp. 581-588, 1993.

[6] M. Faulkner, T. Mattsson, and W. Yates, "Automatic adjustment of quadrature modulators," Electronics Letters, vol. 27, no. 3, pp. 214-216, 1991. 
[7] J. K. Cavers, "New methods for adaptation of quadrature modulators and demodulators in amplifier linearization circuits," IEEE Transactions on Vehicular Technology, vol. 46, no. 3, pp. 707-716, 1997.

[8] R. Marchesani, "Digital precompensation of imperfections in quadrature modulators," IEEE Transactions on Communications, vol. 48, no. 4, pp. 552-556, 2000.

[9] L. Ding, Z. Ma, D. R. Morgan, M. Zierdt, and G. T. Zhou, "Compensation of frequency-dependent gain/phase imbalance in predistortion linearization systems," IEEE Transactions on Circuits and Systems I, vol. 55, no. 1, pp. 390-397, 2008.

[10] D. S. Hilborn, S. P. Stapleton, and J. K. Cavers, "Adaptive direct conversion transmitter," IEEE Transactions on Vehicular Technology, vol. 43, no. 2, pp. 223-233, 1994.

[11] Y.-D. Kim, E.-R. Jeong, and Y. H. Lee, "Joint adaptive compensation for amplifier nonlinearity and quadrature modulation errors," in Proceedings of the IEEE Vehicular Technology Conference (VTC '06), vol. 5, pp. 2290-2293, May 2006.

[12] A. A. Abidi, "Direct-conversion radio transceivers for digital communications," IEEE Journal of Solid-State Circuits , vol. 30, no. 12, pp. 1399-1410, 1995.

[13] M. Valkama, M. Renfors, and V. Koivunen, "Advanced methods for IQ imbalance compensation in communication receivers," IEEE Transactions on Signal Processing, vol. 49, pp. 2335-2344, 2001.

[14] L. Ding, G. T. Zhou, D. R. Morgan, et al., "A robust digital baseband predistorter constructed using memory polynomials," IEEE Transactions on Communications, vol. 52, no. 1, pp. 159-165, 2004.

[15] R. Raich, H. Qian, and G. T. Zhou, "Orthogonal polynomials for power amplifier modeling and predistorter design," IEEE Transactions on Vehicular Technology, vol. 53, no. 5, pp. 14681479, 2004.

[16] R. Raich and G. T. Zhou, "Orthogonal polynomials for complex Gaussian processes," IEEE Transactions on Signal Processing, vol. 52, pp. 2788-2797, 2004.

[17] S. Haykin, Adaptive Filter Theory, Prentice-Hall, Englewood Cliffs, NJ, USA, 2002.

[18] ETSI EN 300744 V1.4.1, "Digital Video Broadcasting (DVB); Frame structure, channel coding and modulation for digital terrestrial television," January 2001.

[19] C. Eun and E. J. Powers, "A new volterra predistorter based on the indirect learning architecture," IEEE Transactions on Signal Processing, vol. 45, no. 1, pp. 223-227, 1997.

[20] M. Saleh, "Frequency-independent and frequency-dependent nonlinear models of TWT amplifiers," IEEE Transactions on Communications, vol. 29, no. 11, pp. 1715-1720, 1981. 